\title{
A Case Report of a Novel Method for the Treatment of Large Osteoarticular Defects Following Shotgun Injury to the Knee
}

\author{
Neal Singleton* and Steve Mc Chesney \\ Orthopaedic Department, New Zealand \\ *Corresponding author: Neal Singleton, Orthopaedic Department, Waikato Hospital, New Zealand

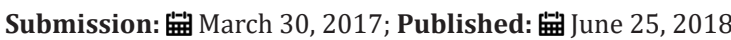

\begin{abstract}
Introduction: Gunshot trauma is increasing worldwide [1]. Consequently, orthopaedic surgeons need to have an understanding of how to treat these often challenging injuries. This report outlines one such case where significant osteoarticular loss made conventional treatment methods difficult and a novel method was utilised.

Case presentation: A 24 year old man presented to our institution having accidentally shot him in the left knee while cleaning a shotgun. He sustained compound distal femoral and proximal tibial fractures with significant osteoarticular loss meaning conventional treatment methods could not be employed. Instead the inner ilium was fashioned to match his missing tibial plateau and a femoral head allograft was used to fill the defect in his medial femoral condyle.
\end{abstract}

Conclusion: Gunshot trauma is common. Due to the high velocity nature bony and soft tissue trauma can be extensive and make conventional treatment methods difficult. This case outlines a method for the treatment of large osteoarticular defects.

Keywords: Osteo articular loss; Novel treatment; Auto graft; Allograft

\section{Introduction}

Gunshot trauma is increasing worldwide with the extremities the most commonly affected anatomic region [1]. Consequently orthopaedic surgeons need to have an understanding of how to

treat these often challenging injuries. This report outlines one such case where significant osteoarticular loss made conventional treatment methods difficult and a novel method was utilised.

\section{Case presentation}

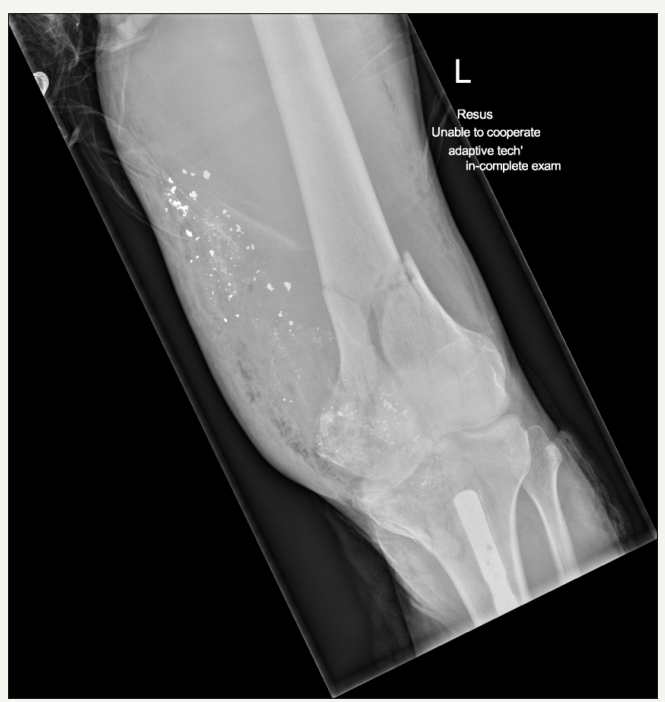

Figure 1: AP X-Ray of knee post-injury. 


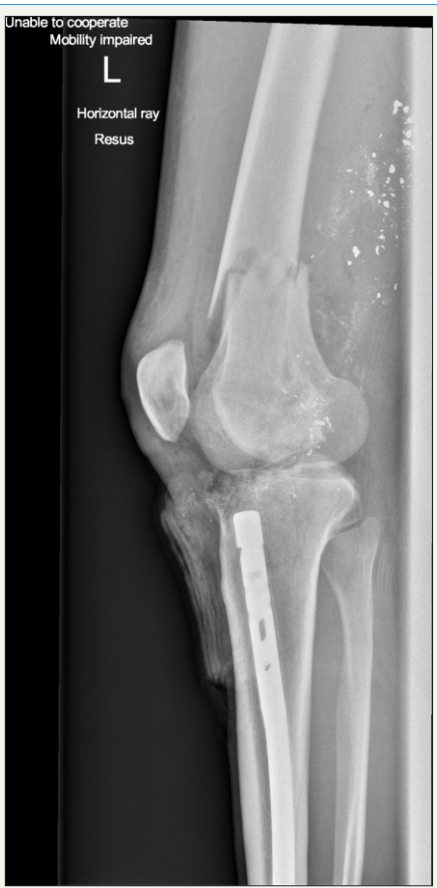

Figure 2: Lateral X-Ray of knee post-injury.

A 24 year old man presented to our hospital having accidentally shot himself in the left knee while cleaning a shotgun. He had sustained compound intra-articular distal femur and proximal tibia fractures with significant bone loss and moderate soft tissue injury (Figure $1 \& 2$ ). There was no associated neurovascular injury. He had an intramedullary tibial nail in situ from a previous tibial shaft fracture. This was an isolated injury and he was otherwise medically well. He underwent debridement, lavage, removal of the tibial nail and spanning external fixation in theatre. 48 hours later he underwent repeat debridement. He was noted to have near complete loss of his tibial plateau (other than poster laterally) and significant loss of the medial femoral condyle. He was treated with intravenous antibiotics and 72 hours after his second washout came forward for definitive surgery. This case report outlines a novel approach for treating complex intra-articular distal femoral and proximal tibial fractures where significant articular bone loss makes standard fixation methods challenging.

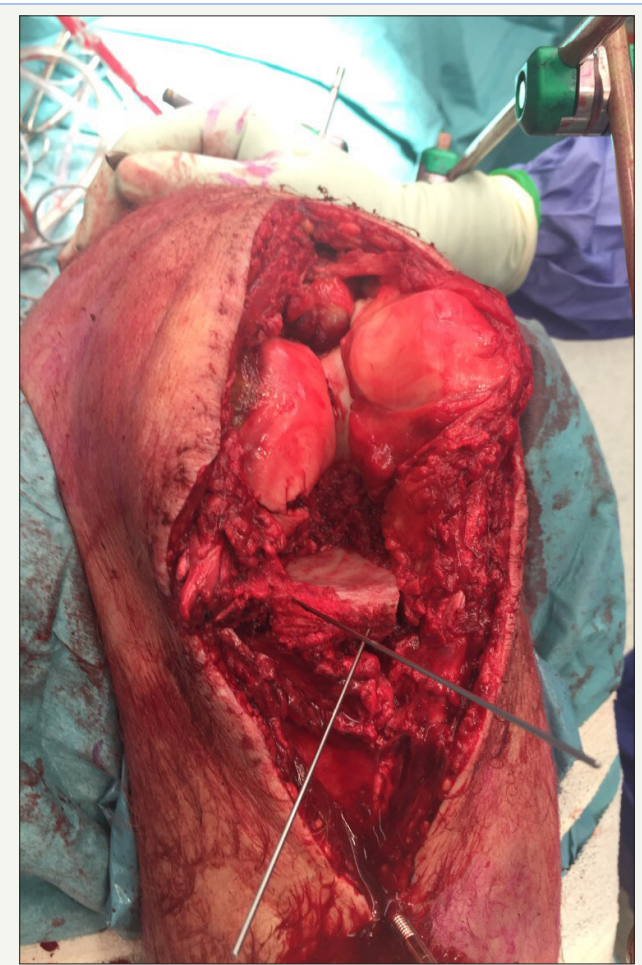

Figure 3: Intra-operative photograph showing reconstruction of tibial plateau using iliac inner table. 
A midline skin incision was made and a standard medial parapatellar approach was utilised. Further debridement of contaminated soft tissue and non-viable bone was performed. Simultaneously, his left iliac crest was approached and a segment of the inner table was excised using an oscillating saw. This measured approximately 5 by $4 \mathrm{~cm}(20 \mathrm{~cm} 2)$ and $5 \mathrm{~mm}$ in depth. This was fashioned to fit the defect in the tibial plateau. The smooth cortical inner surface of ilium was placed cranially facing the joint and created a near horizontal bony platform for femoral articulation (Figure 3).

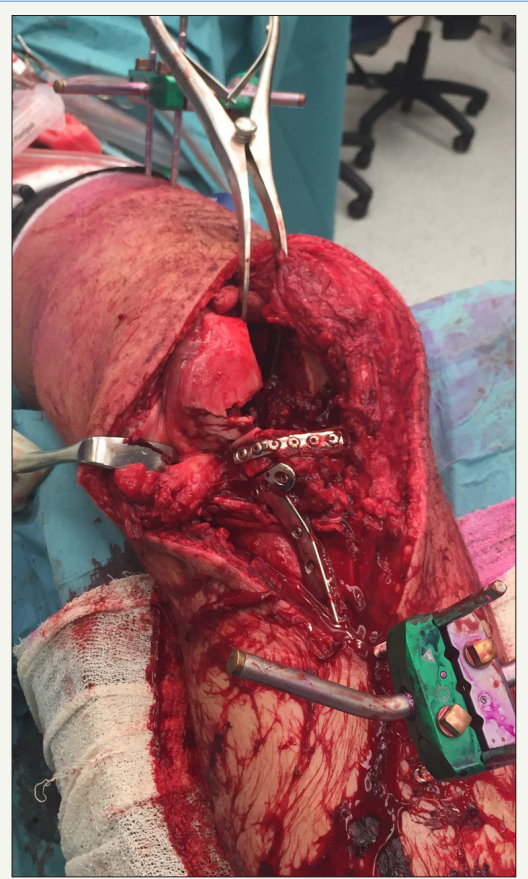

Figure 4: Intra-operative photograph showing iliac inner table secured with a rim plate and further fixation with a locked plate.

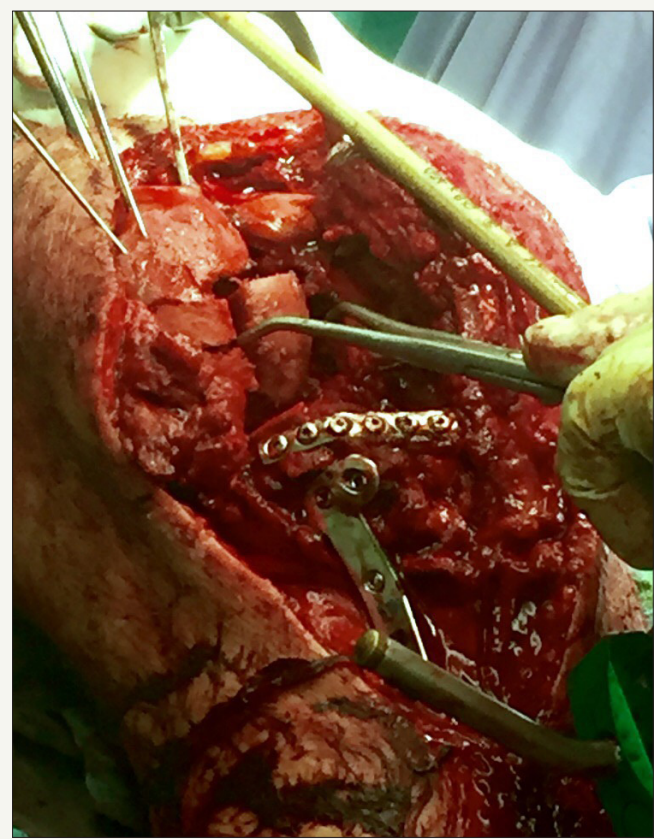

Figure 5: Intra-operative photograph showing fresh frozen allograft femoral head contoured to fill the defect in the lateral aspect of the medial femoral condyle.

This was secured in place using kirschner wires and a rim plate was then used for definitive fixation followed by a standard medial locked tibial plate (Figure 4). A further posterior plate was placed to act as a buttress. The medial femoral condyle was then approached and was similarly non-reconstructable owing to considerable osteoarticular loss (Figure 4) shows the absent medial aspect of the medial femoral condyle). A fresh frozen femoral head allograft was fashioned to replace the lateral part of the missing medial condyle (Figure 5). We chose the surface of the femoral head allograft that had the most well preserved articular cartilage. 
This was temporarily secured using kirschner wires and a rim plate with additional screws outside of the plate was used to definitively fix the allograft to the remaining medial condyle. This restored the spherical shape of the medial femoral condyle and femoral notch and the knee tracked well when taken through a range of motion.

A lateral locking plate was then placed to definitely fix the more proximal extension of the fracture. The patient's ACL and PCL were ruptured but were not reconstructed as it was thought that instability would unlikely be an issue. The wound was primarily closed. The patient was placed into a locked knee brace to facilitate wound healing. At the two week mark the wound was well healed and the brace was unlocked to allow active knee range of motion although the patient was kept strictly non-weight bearing. At most recent follow-up (four months) his knee was stiff with range of motion 10-60 degrees but with no signs of deep infection and all fractures look to have united (Figures 6 \& 7). Although pain free it is likely he will require early knee arthroplasty.

Figure 6: Four month post-operative AP X-Ray showing healed fractures.

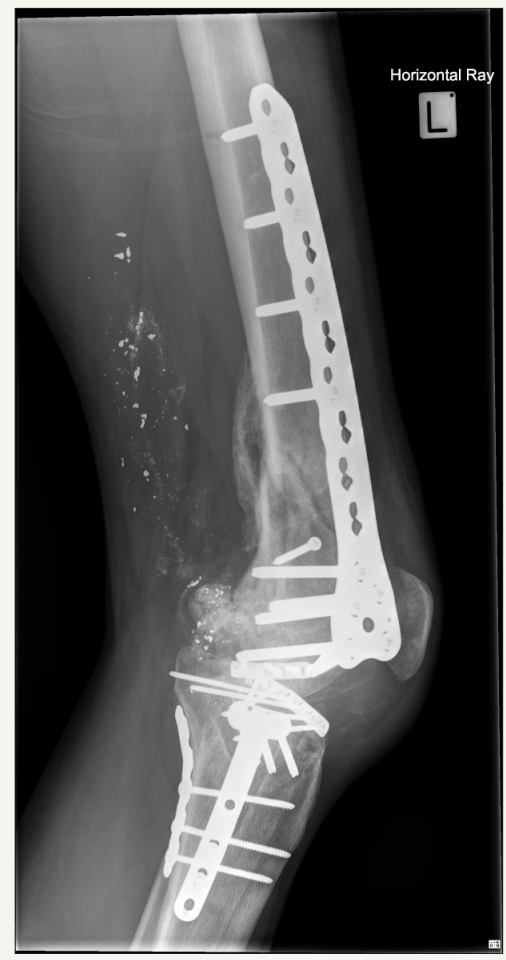

Figure 7: Four month post-operative lateral X-Ray showing healed fractures and fixed flexion deformity of knee. 


\section{Discussion}

The use of iliac inner table auto graft to replace missing tibial plateau although used anecdotally is scarcely reported in the literature. The inner wall of cortical bone would seem a reasonable substitute for missing articular tibial plateau and in theory provides a smooth surface for distal femoral articulation. The hope is that fibrous metaplasia will allow for a substitute articular surface and although not comparable to articular hyaline cartilage may delay the need for joint replacement surgery. Taking only a segment of ilium also keeps donor site morbidity to a minimum and provides auto graft bone with a potentially higher likelihood of union and lower risk of infection. Zhang et al. reported on the use of ilium inner table to repair articular defects in complex tibial plateau fractures [2]. 23 patients with an average age of 28.3 years were followed up for an average of 13.6 months. An average surface area of $6.7 \mathrm{~cm} 2$ of iliac auotograft was used with the concave surface facing cranially. Radiographs showed solid union in all cases and using the Rasmussen score outcomes were excellent in 11 patients, good in 8, fair in 3 and poor in 1.

The use of en bloc femoral osteoarticular allograft to replace missing femoral condyles has previously been reported in the literature both in the setting of tumour resection surgery and trauma with successful outcomes achieved [3-7]. The goal is to maintain knee function with the osteochondral allograft serving as an alternative to arthroplasty. Osteochondral allografts can be fitted and sized to replace large defects. Roffman reported on a 19 year old patient who underwent joint allograft surgery for a traumatic bone defect of the lateral femoral condyle with a good functional result achieved [8]. Hornstein reported on the case of a 15 year old boy who underwent massive frozen osteoarticular lateral femoral condyle allograft following trauma with a good functional outcome at 10 years [6].

In this case, like ours, despite rupture of both the ACL and PCL and obvious laxity on examination Hornstein made no attempt at ligament reconstruction and the patient never reported knee instability. By 10 year follow up the patient was reporting some lateral sided knee discomfort secondary to lateral compartment degenerative changes. Our case differs from most in the literature in that rather than replacing the missing condyle with allograft condyle we used allograft femoral head to replace the missing lateral part of the medial femoral condyle. This was all we had available to us but was able to be shaped to fill the bony defect as well as providing an articular surface which restored the spherical medial femoral condyle and femoral notch.

There are numerous complications with the use of allograft of which infection is potentially the most devastating with a reported incidence of $8 \%$ (Bianchi et al. reported that this incidence is lower for unicondylar reconstructions) [3]. Other potential complications include failure of graft incorporation resulting in non-union and graft subsidence. Early degeneration is considered inevitable in the setting of significant trauma and the use of osteoarticular allo grafts. Bianchi reported that in 10 out of 12 cases degeneration occurred even when the ACL was reconstructed [3]. Risk of disease transmission is low and similar to that of a blood transfusion and in retrieval studies no evidence of transplant rejection was encountered [9].

The advantage of this procedure includes the maintenance of bone stock which keeps open options for future surgery. Allografts provide a source of chondrocytes within an existing extracellular matrix and have been shown to be viable long-term [10]. Used in the setting of trauma, allografts have been shown to have better outcomes than when used for degenerative diseases [11]. Single sided allografts (on either the femoral or tibial side of the joint) have been shown to have better results than when used on both sides of the joint [12].

Alternative treatment strategies in this case could have included a knee arthrodesis although knee arthodeses are known to be poorly tolerated particularly in younger active patients. Primary knee arthroplasty was not deemed suitable given the degree of trauma and the potential intra- and post-operative complications. A knee arthroplasty in this setting would also have required augments to replace missing bone, long stem prostheses given the extent of the proximal femoral and distal tibial fracture lines and would carry a high risk of infection and stiffness. Longevity of the implant could also potentially be considered a contraindication to its use in our 24 year old patient. Spanning external fixation (articulating or nonarticulating) would not have been suitable in this case given the degree of bone loss on both sides of the joint.

\section{Conclusion}

In conclusion, this case has reported on a challenging scenario where significant osteoarticular loss on either side of the knee joint precluded standard fixation methods and instead the use of a novel technique where a large fragment of inner ilium table was used as auto graft to reconstruct the tibial plateau and a frozen femoral head allograft was used to reconstruct missing femoral condyle. The knee joint was reasonably well reconstructed with good on table range of motion. Although stiff the patient is now pain free and all fractures have untied with no evidence of infection or graft subsidence.

\section{Clinical Message}

Gunshot trauma is not uncommon and is increasing in orthopaedic practise. There can be significant osteochondral as well as soft tissue injuries precluding standard treatment methods. This case report has outlined how we dealt with a particularly challenging scenario of missing tibial plateau and femoral condyle using the patient's own inner ilium as well as an allograft femoral head with pleasing results.

\section{References}

1. Dougherty P, Najibi S, Silverton C, Vaidya R (2009) Gunshot wounds: Epidemiology, wound ballistics, and soft-tissue treatment. Instr Course Lect 58: 131-139.

2. Zhang J, Zhang W, Shang B, Du F, Zhou E, et al. (2008) Taking ilium inner table to repair the articular surface defects of complex tibial plateau. Zhongguo Gu Shang 21(2): 116-117. 
3. Bianchi G, Staals E, Donati D, Mercuri M (2009) The use of unicondylar osteoarticular allografts in reconstructions around the knee. Knee 16(1): $1-5$.

4. Musculo D, Ayerza M, Aponte T, Farfalli G (2007) Unicondylar osteoarticular allografts of the knee. J Bone Joint Surg Am 89(10): 21372142.

5. Lee J, Wans S, Choi H, Hwang B, Lim Y, et al. (2011) Unicondylar osteoarticular allograft reconstruction of the distal femur in a patient with a traumatic osteoarticular defect of the lateral femoral condyle. Knee Surg Sports Traumatol Arthrosc 19(4): 556-558.

6. Hornstein S, Moukoko D, Deroussen F, Plancq M, Collet L, et al. (2015) Successful hemicondylar femoral allograft for traumatic bone loss: a paediatric case study with ten years of follow-up. Knee 22(1): 63-66.

7. Taylor D, Bohm K, Gross A (2011) Use of fresh osteochondral allograft in repair of distal femur after trauma. Mcgill J Med 13(1): 22.
8. Roffman M (1986) Combined auto- and alloosteochondral grafts for large defects. J Trauma 26(2): 189-191.

9. Buck B, Malinin T, Brown M (1989) Bone transplantation and human immunodeficiency virus. An estimate of risk of acquired immunodeficiency syndrome (AIDS). Clin Orthop Relat Res 240: 129136.

10. Convery F, Akeson W, Amiel D, Meyers M, Monosov A (1996) Longterm survival of chondrocytes in an osteochondral articular cartilage allograft. A case report. J Bone Joint Surg Am 78(7): 1082-1088.

11. Convery F, Meyers M, Akeson W (1991) Fresh osteochondral allografting of the femoral condyle. Clin Orthop Relat Res 273: 139-145.

12. Chu C, Convery F, Akeson W, Meyers M, Amiel D (1999) Articular cartilage transplantation. Clinical results in the knee. Clin Orthop Relat Res 360: 159-168.
Creative Commons Attribution 4.0

International License

For possible submissions Click Here

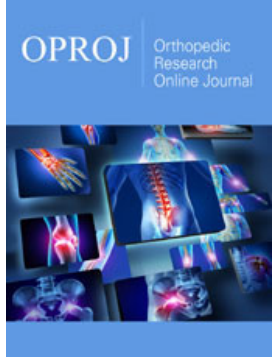

Orthopedic Research Online Journal

\section{Benefits of Publishing with us}

- High-level peer review and editorial services

- Freely accessible online immediately upon publication

- Authors retain the copyright to their work

- Licensing it under a Creative Commons license

- Visibility through different online platforms 